\title{
THE INFLUENCE OF ENVIRONMENTAL FACTORS ON NONALCOHOLIC FATTY LIVER DISEASE AND OBESITY
}

\section{Kvit Khrystyna ${ }^{1}$ \\ Kharchenko Viacheslav ${ }^{2}$}

DOI: http://dx.doi.org/10.30525/978-9934-571-31-2_6

\begin{abstract}
Non-alcoholic fatty liver disease (NAFLD) is the most common kind of liver injury in the world and its prevalence is due to the lack of "in-time" diagnosis and, as a consequence, treatment. A lot of different diseases are complicated by the fatty liver infiltration, the most often is obesity. The progress of the disease could depend on the environmental factors and the genetic disabilities. The aim of the review article was to analyze the latest data about the factors which impact on the NAFLD development in obese patients, including the gut microbiota, genetic predictors and environmental components, to find the new ways of pathogenesis, diagnosis and treatment. Non-alcoholic liver steatosis is diagnosed in $70 \%$ of obese patients (although some authors report a significantly higher (up to 95\%) incidence of cases) and $35 \%$ of thin patients, and non-alcoholic steatohepatitis (NASH) - in $18.5 \%$ of obese patients and $2.7 \%$ - with insufficient body weight. Among patients with severe obesity and BMI more than $35 \mathrm{~kg} / \mathrm{m} 2$, the prevalence of NAFLD and NASH is $91 \%$ and $37 \%$, respectively. The combination of facts, available at the moment, suggests that one of the central role in the NAFLD development plays disturbance of intestinal microbiota and its permeability. Intestinal microflora can affect the NAFLD due to three mechanisms: 1) an increase in ethanol production in the intestinal cavity; 2) metabolic disorders of food choline (necessary for the synthesis of very low-density lipoproteins and elimination of liver lipids); 3 ) the release of bacterial lipopolysaccharides. The diagnostic methods, which allow investigation of fatty liver infiltration in the early stages, are considered. Despite the lower sensitivity and specificity compared with CT, ultrasound is considered as an acceptable first-line procedure for diagnostic NAFLD in clinical practice. The waist cir-
\end{abstract}

\footnotetext{
${ }^{1}$ Ph.D, Associate Professor,

Danylo Halytsky Lviv National Medical University, Ukraine

${ }^{2} \mathrm{Ph} . \mathrm{D}$, Associate Professor,

Shupyk National Medical Academy of Postgraduate Education, Ukraine
} 
cumference, the liver obesity index (IOP), and lipid accumulation products is closely related to the IP, indicate liver steatosis and may be useful for large screening among the general population instead of ultrasound. At present, there is no specific therapy for NAFLD in combination with obesity. Diet modulation and life style modification are the important part of treatment. Given the fact that the key mechanism in the progress of NAFLD and obesity is the IR, the use of pharmacological agents that have a positive effect on the sensitivity of tissues to insulin, - insulin sensitizers, is confirmed. Taking into account, that there are no specific drugs for the NAFLD treatment, the study of the intestinal microbiota influence on the pathogenesis and progress of this disease may have new therapeutic perspectives. In particular, the modulation of the intestinal microbiota can be achieved by the use of prebiotics, probiotics, selective intestinal antibiotics (rifaximin), and fecal microbiota transplantation.

\section{Introduction}

Nonalcoholic Fatty Liver Disease (NAFLD) is a chronic metabolic, stress-induced liver disease associated with insulin resistance in patients who do not consume alcohol in hepatotoxic doses (more than $40 \mathrm{~g}$ of pure ethanol for men and $20 \mathrm{~g}$ for women per day). Today NAFLD becomes one of the most common chronic liver diseases around the world. The modern notion of NAFLD includes the range of liver lesions that are differed by clinical and histological changes: steatosis, steatohepatitis, and fibrosis. Liver steatosis, usually, has a benign course and does not correlate with mortality, whereas progression of this disease to non-alcoholic steatohepatitis (NASH) increases the risk of forming liver cirrhosis and liver failure [6].

Population surveys suggest that $60-80 \%$ of cryptogenic liver cirrhosis is a consequence of NASH. Since NASH's term was first proposed by J. Ludwig in 1980, the prevalence of NAFLD has increased in parallel with the dramatic obesity and diabetes increase in the population, and is now the most common cause of liver disease in the world. NAFLD is closely linked to insulin resistance (IR), obesity and is recognized as a liver manifestation of metabolic syndrome (MS). The association of NAFLD with obesity, especially abdominal, metabolic syndrome and various metabolic markers of the risk of developing cardiovascular diseases and type 2 diabetes mellitus (DM type 2) directly affects the prognosis of the underlying diseases, as well as the life duration of obese patients [11]. 
Patients with NAFLD have a higher prevalence of cardiovascular disease (CVD) than patients without this pathology, regardless of the severity and traditional risk factors. Thus, mortality from CVD in comparison with mortality from liver diseases in the population is 7.5 and $0.2 \%$, respectively. And in persons with steatosis and NASH, these rates increase by 4 or more times, while the mortality rate of the CVD in the group of NASH predominates over mortality from liver disease and is $36 \%$ versus $6.8 \%$, respectively [15].

Prevalence of NAFLD. Since the verification of the NAFLD diagnosis requires histological confirmation, the data on its prevalence are significantly different and in many cases may not correspond to reality. In the United States, the prevalence of NAFLD among liver diseases has risen to the first place and is $20-30 \%$. In the presence of several etiological factors above, the risk of morbidity is almost $100 \%$. The prevalence of NASH among patients with persistent signs of cytolysis of "unknown origin" is $20-32 \%$. The frequency of the discovery of NAFLD in western countries among patients treated with liver biopsy is $7-9 \%$. Up to $40 \%$ of non-viral cirrhosis is associated with NASH, whose decompensation has led to 5-8\% of liver transplantations in the United States and the EU. The prevalence of NAFLD against the background of the IR syndrome on a global scale may be up to $10 \%$ (near 600 million people). NASH most often occurs against the backdrop of a progressive course of diabetes type $2(88 \%)$ and metabolic syndrome $-81 \%$ of cases $[6 ; 19]$.

NAFLD is associated with some chronic non-infectious diseases that are considered within the framework of metabolic syndrome (MS), the prevalence of which has now reached the scale of the epidemic. To date, about 1.46 billion adults (over 15 years old) worldwide have overweight or obese. Over the last quarter century, the number of patients with severe forms of obesity and type 2 diabetes doubled. NAFLD in obese patients is detected in 4.6 times often than in the population. Non-alcoholic liver steatosis is diagnosed in $70 \%$ of obese patients (although some authors report a significantly higher (up to $95 \%$ ) incidence of cases) and $35 \%$ of thin patients, and NASH - in $18.5 \%$ of obese patients and $2.7 \%$ - with insufficient body weight. According to other data, NASH is in $60-80 \%$ of obese patients $[11 ; 19]$.

At the same time, up to $25 \%$ of NAFLD patients may not have obesity, but have laboratory and instrumental evidence of liver changes in the type 
of fatty liver. In a recent study, it was reported that among patients with NAFLD without DM, $22 \%$ were thin, $64 \%$ had minimum of criteria for MS, $12 \%$ had not found any criteria for MS. Korean scientists have shown that liver steatosis develops in patients with an increase in body weight, but not necessarily accompanied by an increase in the body mass index. Steatosis, in the opinion of researchers, is formed during the process of weight gain, regardless of whether BMI is normal $[13 ; 20]$.

While alcohol fatty liver disease is observed predominantly in men, among women with NAFLD, women predominate (65-83\%). In most of them, excess body weight, which is $10-40 \%$ higher than normal, and $25-75 \%$ - DM type 2. Hyperlipidemia (type II or IV) is diagnosed in $20 \%$ of patients, and in the presence of obesity - more than $50 \%$. The average age of patients at the time of diagnosis of NASH is 50 years.

The results of T. Poynard and V. Ratziu (2002) analysis of statistical data to determine the location of NASH in the structure of morbidity and prevalence of major liver diseases in the world population for the first time demonstrated the same prevalence of ASH and NASH, which in both cases is $10 \%$ (600 million people), while chronic HBV-hepatitis is detected in 5\% of the population (300 million people), chronic HCV-hepatitis - in 3\% (180 million people), and hemochromatosis - in $0.5 \%$ (30 million people). Since today there is no specific data on the frequency of cirrhosis development due to NASH, taking into account the frequency of cryptogenic cirrhosis - about 5-15\%, we can assume that NASH occupies a significant part of this etiological niche. In general, in Ukraine for five years, the incidence of steatohepatitis of different etiologies has increased to $76.6 \%$, cirrhosis $-75.6 \%$, the prevalence of NASH during this period - increased in $2.2[6 ; 8 ; 11]$.

Data from 2016 were able to obtain more detailed results on the prevalence of NAFLD using a variety of diagnostic techniques, since this pathology often has an asymptomatic duration with normal liver parameters, which significantly complicates the evaluation of its prevalence, and diagnosis of NAFLD by ultrasound can be considered reliable only when steatosis injures more than a third of the liver. Taking into account these facts, the prevalence of NAFLD is $20-30 \%$ of the adult population in the western and $15 \%$ in the asian countries. With the implementation of informative diagnostic methods such as magnetic resonance imaging and spectroscopy (MRI, MRS), the proportion of NAFLD is $34 \%$ of adult americans [11]. 
Among patients with severe obesity and BMI more than $35 \mathrm{~kg} / \mathrm{m} 2$, the prevalence of NAFLD and NASH is $91 \%$ and $37 \%$, respectively. In the United States, almost $40 \%$ of the first identified cases of the chronic liver disease are associated with NAFLD. The unexpectedly high prevalence of histologically verified NAFLD was obtained in the study of healthy liver donors (12-18\% in Europe, 27-38\% in the United States).

Recent studies conducted in highly specialized centers of care showed a high prevalence of histologically verified NASH in NAFLD patients: $43-55 \%$ in patients with elevated aminotransferase levels, $49 \%$ of patients with morbid obesity, and $67 \%$ among patients with the chronic liver disease. The incidence of primary NAFLD in Italy is estimated at $2 / 100 /$ year, while Japanese studies in selected populations summed indicator 10/100/ year. For comparison: for secondary NASH, due to the use of tamoxifen, the predicted level is $0.2 / 100$ women/year [19].

In contrast of alcohol steatohepatitis, short-term prognosis with NAFLD is favorable. Long-term prognosis depends on histological data at the time of the study. In the $12-40 \%$ of patients with steatosis $8-13$ years old, NASH with early fibrosis develops. Among these patients during the same period, about $25 \%$ develop cirrhosis or hepatic decompensation $(15 \%)$ or pre-cirrhotic changes (10\%). Approximately, in $7 \%$ of patients with compensated cirrhosis due NAFLD/NASH after ten years hepatocellular carcinoma (HCC) develops, about $50 \%$ of them require a liver transplant or die due to the liver complications. The risk of developing HCC in cirrhosis after NAFLD/ NASH can be compared with those with alcoholic cirrhosis or cirrhosis due to hepatitis C. Today about $12 \%$ of all liver transplants in the United States are performed on cirrhosis due NAFLD/NASH. The average life expectancy of patients with NAFLD is lower than in the population. If in the general population hepatic pathology is only the 13th leading cause of death, but in patients with NAFLD/ NASH - 3rd cause [8].

Etiology of NAFLD. The cause of the disease can be genetic factors (birth defects $\beta$-oxidation, Wolman's disease, deficiency of $\alpha 1$-antitrypsin). NAFLD is also associated with inflammatory intestinal diseases, malabsorption syndrome, surgical interventions on the small intestine. One of the possible reasons for the development of the inflammatory component in liver steatosis is endotoxemia, which is associated with excess bacterial proliferation in the small intestine. Exogenous factors of NAFLD include prolonged parenteral nutrition, unbalanced fat, and carbohydrate, lack of 
protein in the diet, fasting, hypercaloric diet with excess carbohydrates, rapid weight loss. Development of steatosis after the application of certain drugs (amiodarone, tamoxifen, nifedipine, diltiazem, synthetic estrogens, high doses of glucocorticosteroids) has been noted [4].

The leading role in the pathogenesis of NAFLD belongs to the IR, resulting in the accumulation of triglycerides, free fatty acids (FFA) in the liver, oxidative stress develops, steatohepatitis is formed. FFAs is released from visceral adipose tissue (VAT), which includes mesentery and omentum and contains mature adipocytes, preadipocytes, endothelial cells, fibroblasts, macrophages, granulocytes. Utilization of FFAs derived from abdominal fat occurs in hepatocytes as a result of $\beta$-oxidation in mitochondria and gluconeogenesis. The elevated SREBP-1 expression (a factor that activates the expression of all genes involved in the lipogenesis process), which is observed with excessive FFAs and hyperinsulinemia, leads to hypertrophy of adipocytes in the adipose tissue, with subsequent release of FFAs, and in hepatocytes - to intensify the synthesis of FACoA (fatty acyl-coenzyme A active intracellular form of fatty acids. The latter is transformed into TG or very low-density lipoprotein (VLDL), and, in the end, the above cascade of pathological reactions stimulates the development of steatohepatitis. Under conditions of excess energy or hyperglycemia, excessive glucose activation CREBP (a carbohydrate responsive element-binding protein), which is responsible for binding to glucose) is observed, which leads to stimulation of the liver pyruvatekinase and, consequently, to the increased formation of acetyl CoA, which is used for the synthesis of LCD [18].

The peroxisome proliferator-activated receptor (PPARs), which is involved in lipid metabolism, glucose, inflammation and tumor growth, differentiation of adipocytes, and modulation of peripheral tissues of the peripheral tissues plays an important role in lipogenesis. All three types of PPARs receptors $(\alpha, \beta / \delta, \gamma)$ are bound and activated by HF. PPARs direct the transcription of a large number of genes, including coding mitochondrial, peroxisomal, and some microsomal enzymes of liver metabolism in the liver. Also, PPARs control the genes responsible for transport and absorption of LC, modulating the metabolism of TP enriched lipoproteins [18].

In the pathogenesis of NAFLD, bile acids play a role, which, besides participating in the absorption of fats and maintaining cholesterol homeostasis, act as biological signaling molecules. The bile acids control their synthesis in the liver by reverse inhibition of the nuclear X-receptor. The 
bile acids suppress production in the liver TG and VLDL by activating the signaling pathways of the same nuclear receptors. The signaling role of bile acids is also manifested in the fact that they increase energy expenditure by increasing the content of triiodothyronine in brown fat tissue and skeletal muscle, inhibiting the development of obesity and IR.

Obesity increases FFA intake into the liver, which stimulates the steatosis development and occurs FFA oxidation, which forms the products of lipid peroxidation (LPO) and reactive forms of oxygen. Oxidative stress develops, which accompanies by the transformation of steatosis into the steatohepatitis. Factors, which cause inflammation, are lipophilicity, impaired function of intracellular structures (mitochondria, microsomes). The factors that provoke the hepatocytes destruction and the development of fibrosis are peroxide lipid oxidation (PLO), secretion of cytokines.

It is known that not only insulin resistance and obesity play a role in the development of NAFLD, but also violation or hypersecretion of visceral adipose tissue adipocytokines. Visceral fatty tissue which participates in the progress of the visceral obesity, in contrast to adipose tissue of another localization, is richer in innervations, vascularized and is directly associated with the portal system. Adipocytes of visceral adipose tissue, possessing high sensitivity to the lipolytic action of catecholamines and low sensitivity to the antilipolytic action of insulin, contribute to the development of hyperlipidemia, hyperinsulinemia, and insulin resistance $[4 ; 5 ; 17]$.

One of the products of adipocytes is CNF- $\alpha$, which stimulates the development of IP. Leptin and resistin are also relevant to the accumulation of fat in the visceral tissue. Leptin has an anti-static effect on the hepatic tissue. Mechanisms of participation of leptin in excessive deposition of lipids in hepatic tissue and triggering fibrogenesis were studied. Leptin enhances phagocytic activity and the production of cytokines by Kupffer cells and macrophages, stimulates the proliferation of endothelial cells and their production of active forms of oxygen, that is, it participates in the launch of the second push (oxidative stress) in the pathogenesis of NAFLD. It can be considered as a predictor of the development of steatosis, inflammatory changes in the liver tissue, fibrosis with NAFLD, but the results of these studies are controversial. It was found that in subjects with abdominal obesity the level of leptin is higher than that of people without signs of obesity, and in some of them the level of leptin does not exceed normal, that is, in patients with excess body weight and obesity, there is a phenomenon of hyperleptinemia and leptin resistance [2]. 
The leptin influence is realized at the level of the hypothalamus, where it binds to the receptors, thereby causing the activation of signals that inhibit the desire to eat and increase energy expenditure. With obesity, the compensatory resistance of the hypothalamus may arise to the central action of leptin, which in turn leads to hyperleptinemia through the feedback mechanism. Also, the ability of leptin to stimulate cellular immune response and to influence the production of proinflammatory cytokines has been proven [14].

The natural antagonist of the leptin effects is adiponectin, a collagen-like protein synthesized by adipocytes, capable of regulating energy homeostasis and causing anti-inflammatory and antiatherogenic effects. The level of adiponectin decreases with obesity. Hypoadiponectinemia along with an increase in the level of TNF- $\alpha$ and other proinflammatory factors may be predictors of cardiomaterial disorders.

It has been noticed that in patients with steatohepatitis, the level of adiponectin in plasma is lower than in the control group and in people with plain steatosis, regardless of the IR and body mass index (BMI). In the inflammatory process, adiponectin secretion decreases. It is hypothesized that hypoadiponectinemia is also involved in the development of dysfunction of the pancreatic $\beta$-cells, which is observed in NAFLD. In contrast to the statement on the hepatoprotective effect of adiponectin, there is evidence that the level of circulating adiponectin is elevated in liver cirrhosis, both in experimental animals and in humans [8].

According to some authors, the indicator, which is closely related to the manifestations of excess body weight and levels of carbohydrate and lipid metabolism, is the ratio of leptin/adiponectin. Given the BMI, this correlation correlates with the presence or absence of MS patients and is used as a biochemical marker for the risk of developing an IR.

The combination of facts, available at the moment, suggests that one of the central roles in the development of NAFLD play disturbances of intestinal microbiota and intestinal permeability. Intestinal microflora can affect the development of NASH due to three mechanisms: 1) an increase in ethanol production in the intestinal cavity; 2) metabolic disorders of food choline (necessary for the synthesis of very low-density lipoproteins and elimination of liver lipids); 3 ) the release of bacterial lipopolysaccharides. New experimental and clinical findings have led to a better understanding of the multiple factors that determine the emergence of $\mathrm{NASH}$, which is 
most likely a result of the combined action of genetic, social, behavioral factors and environmental factors. Recent studies tend to suggest that liver steatosis in most patients has a benign course, and NASH may be a separate disease with different pathogenesis. Many factors, especially those associated with intestines or adipose tissue, can be affected in parallel, which ultimately leads to inflammation of the liver.

Complement knowledge and genetic mechanisms of development of NAFLD [16].

As it became known, in the pathogenesis of steatosis, an important role is played by miRNA-10b (miRNA-10b), which regulates the differentiation of adipocytes, lipid metabolism, glucose-mediated insulin secretion. It should also be mentioned the polymorphism of the genes ENPP1 / PC-1 Lys121 GLN and IRS-1 Gly972Arg, which is detected in 28.7 and $18.1 \%$ of the patients, respectively, and is associated with obesity, dyslipidemia, and the risk of diabetes, since the present polymorphism plays a role in the activation of the substrate insulin-1 receptor, that is, in the formation of insulin resistance. In addition to the above genes, the pathogenesis of NAFLD also has the significance of the polymorphism of the gene PNPLA3. This gene encodes the protein synthesis of adiponitrile. It is known that the level of mRNA PNPLA3 increases in adipocytes and hepatocytes in obese patients. Increased activity of the product PNPLA3 does not correlate with insulin resistance, and it can be assumed that the effect of adiponitrile leads to an increase in the concentration of triglycerides in the liver tissue [8].

Factors, which influence on the NAFLD. Acknowledged risk factors for NAFLD are obesity and severe weight loss. Age, gender, and ethnicity are also reliably related to NAFLD. It has been established that the risk of progression of NAFLD in fibrosis and cirrhosis increases with age, and the overall prevalence of diseases is higher among men belonging to the African-American ethnic group, Latin America, and Hispanic ethnicity. This difference can be explained by the existence of an ethnic difference in lipid metabolism. Moreover, the risk factor for NAFLD is female.

Also, the risk factors in patients with NAFLD are arterial hypertension (AG) (69.9\%), abdominal obesity (56.2\%), hypercholesterolemia (68.8\%). Without a doubt, there are many other factors (some medications, rapid weight loss, prolonged parenteral diet, lipodystrophy, Wilson-Konovalov's disease, hemochromatosis, hepatitis $\mathrm{C}$, etc.). However, the main role in the development of NAFLD belongs to insulin resistance and obesity [12]. 
Moreover, Yoneda et al. reported that the detection frequency of P. gingivalis in the saliva of patients with NAFLD and NASH was significantly higher than that of non-NAFLD control subjects. Also, they presented preliminary findings showing that non-surgical periodontal treatments in 10 patients with NAFLD for three months improved liver function parameters such as serum levels of AST and ALT. Taking all of this into account, it is thought that infection with periodontal pathogens mainly composed of $\mathrm{P}$. gingivalis is associated with fibrosis severity in patients with NAFLD and that the prevention and eradication of $\mathrm{P}$. gingivalis infection through periodontal treatment may have a beneficial effect upon NASH [21].

The factors of progression of the disease today include the age more than 45 years, obesity (body mass index greater than 30), type 2 diabetes, hypertension, hypertriglyceridemia, ALT to AST ratio greater than 1, $\mathrm{HCV}$ infection. Potential external causes of NAFLD progression include dietary factors (a high-fat diet, meat, sweet drinks, low antioxidants and omega-3 fatty acids) and intestinal dysbiosis, including excessive bacterial growth in the small intestine. Genetic factors are likely to play a significant role in enhancing susceptibility to NAFLD, as evidenced by family cases and inter-ethnic variations. The presence in the family of at least one person with NAFLD forms an increased risk for the development of the disease, regardless of age and body mass index. It is assumed that polymorphisms of genes encoding microsomal transfer superoxide dismutase 2, endotoxin receptor CD14, TNF- $\alpha$, TGF- $\beta$ and angiotensinogen increases the risk of developing NASH and fibrosis [13].

Clinical diagnosis. BMI is a simple, reliable screening criterion for assessing normal or overweight and obesity. At the same time, research in recent years has shown that BMI is not a sufficient criterion for predicting the development and course of all diseases associated with obesity and excess body mass. It was ammounced in the 23rd Annual Scientific Congress in 2014, where the American Association of Endocrinologists considered a new algorithm for diagnosis of obesity, which includes two components: a) BMI assessment with an ethnicity correction to identify individuals with high levels of adipose tissue and b.) The presence and severity of complications associated with obesity. It is even proposed to consider changing the term "obesity" (for example, on "adiposity-based chronic disease" (ABCD) [2].

In order to suspect a patient at the initial examination, the doctor should first of all rely on knowledge of the risk factors for the formation of liver ste- 
atosis (age over 45-50 years of age, female sex, obesity or overweight, and other manifestations of metabolic syndrome (hypertension, CD2, hyperlipidemia and hypertriglyceridemia) An important point in the diagnosis of NAFLD is the careful studying of anamnesis to exclude the alcohol impact on the liver. It is also necessary to assess the lifestyle, food habits, the level of physical activity of the patient, the presence of metabolic disorders and liver diseases in the family, as it is known that such factors as the presence of viral lesion of the liver (especially the genotype 3 of the viral hepatitis C), parenteral nutrition, rapid weight loss (more than $10 \%$ of the available per year), Wilson- disease, hemochromatosis, congenital metabolic disorders, Reye's syndrome, HELLP syndrome - can lead such changes in the liver parenchyma as fatty liver (so-called "secondary steatosis") [8].

It is important to mention the influence of medicinal substances on the formation of liver steatosis. Medications can cause small-drops or largescale obesity of hepatocytes. Medicines, which can cause such changes are acetylsalicylic acid, non-steroidal anti-inflammatory drugs, valproic acid, tetracycline, antiviral drugs used in the treatment of HIV infection, glucocorticoid hormones and methotrexate. In turn, steatohepatitis may be associated with the use of perhexiline maleate, amiodarone, synthetic estrogens, calcium antagonists, antimalaria agents. Prescribtion of the above drugs should be excluded in patients with NAFLD [12].

NAFLD is characterized by the asymptomatic duration. Patients with fatty infiltration have no complaints, or their complaints are nonspecific: weakness, fatigue, discomfort, and heaviness in the right hypochondrium. At physical examination in 50-75\% of patients with NAFLD detect hepatomegaly. Liver steatosis is detected accidentally by ultrasound, which was done for other reasons. Ultrasound is a very common method of diagnosis of NAFLD, in which steatosis appears as a heterogenous liver, but does not indicate the amount of fat [18].

Despite the lower sensitivity and specificity compared with computer tomography (CT), ultrasound is considered as an acceptable first-line procedure for diagnostic NAFLD in clinical practice. MRI determine the level of steatosis reliably, but it is limited by problems of standardization and high cost. In separate groups of patients with NAFLD diagnostically justified is the definition of the stiffness of the liver by conducting transient elastography. However, the limit values of fibrosis are not always correlated with the data of the biopsy; their size is influenced by the presence of steato- 
sis and inflammation, which can increase the stiffness of the liver. The main predictor of inadequate informativeness of transient elastography is the body mass index $(25 \%>30 \mathrm{~kg} / \mathrm{m} 2,41 \%>35 \mathrm{~kg} / \mathrm{m} 2)$. The sensitivity and specificity of the ultrasound examination is $60-94 \%$ and $88-95 \%$, respectively, and decreases with the increase in BMI and the degree of steatosis to 49 and $75 \%$. Quantify the degree of severity of fatty infiltration in the liver allows a magnetic resonance imaging with contrast. Elastography (Fibroscan), which determines the density and presence of liver fibrosis with the help of elastic waves, is not informative at BMI greater than 30, that is, in obesity, and does not allow to differentiate steatosis from steatohepatitis [5].

Patients with NAFLD can be diagnosed by increasing of such laboratory parameters (ALT, AST, gammaglutamyltranspeptidase - GGTP, alkaline phosphatase), which could not determ the stage and activity of the process. Elevated levels of serum transaminases are found in $20-21 \%$ of patients with NAFLD. However, the average performance of aminotransferases does not exclude the probability of necrotic-inflammatory changes and liver fibrosis. According to studies, at NASH, ALT levels are more likely to be higher than AST levels, and lowered ALT combined with severe obesity can be evidence of severe fibrosis and cirrhosis of the liver. In some cases, patients with NAFLD have iron metabolism disturbances (increased levels of ferritin and saturation of serum transferrin), not associated with the presence of hereditary hemochromatosis. It is also possible to identify various components of the metabolic syndrome: increase triglycerides, decrease in HDL in the blood, damage to carbohydrate metabolism [18].

Regarding non-invasive research methods, they can indicate the mass of liver tissue affected by fibrosis and the presence of NASH that is commonly found together. Focusing on fibrosis is based on the results of many studies that demonstrate that fibrosis, and not inflammation, is more prognostically significant. Definition and monitoring of liver fibrosis (LF) are one of the most important aspects in the management of patients with NAFLD. According to modern notions, serologic markers of LF are divided into lines reflecting the metabolism of the extracellular matrix, and indirect, which indicate a violation of the function of the liver in the expressed LF or cirrhosis $[1 ; 4 ; 8 ; 15]$.

Biomarkers of fibrosis are conventionally divided into direct and indirect. Direct biomarkers of fibrosis are fragments of cellular matrix of the liver, formed by cells, and molecules that participate in the regulation of 
progression and regression of fibrosis. It is hyaluronic acid (HA), collagen IV and VI, the aminoterminal fragment of procollagen III (P3NP), matrix metalloproteinases, and TIMP-1, a tissue inhibitor of metalloproteinase. Indirect markers of fibrosis are molecules released into the blood due to the inflammatory process going on in liver, in particular, aminotransferases (ALT and AST), molecules synthesized, regulated or secreted by the liver, for example, apolipoprotein A1, alpha-2-macroglobulin (A2M), ferritin, haptoglobin, coagulation factors, cholesterol and bilirubin, and markers of pathologies, which occur when damaged no liver function, such as insulin resistance $[1 ; 3 ; 6]$.

For clinical practice, various estimates of prognostic indices for assessing the severity of liver fibrosis according to the indirect markers: APRI, ELF, NAFLD Fibrosis Score, FIB-4, FibroFast, FibroIndex, FibroMeter, FPI, Forns, GUCI, Hepascore, HALT-C, MDA, PGA, PGAA.

The system of non-invasive diagnostic of liver, FibroMax and SteatoScreen ("BioPredictive,» France), was officially recognized as an alternative to liver biopsy in 2007. It displays the stages of fibrosis (F0-F4) and the degree of the necrotic-inflammatory process in the international METAVIR system, and also provides an quantitative and qualitative assessment of fibrosis, liver steatosis in all stages, regardless of localization. The American Association of Gastroenterologists, Association for the Study of Liver Diseases recommended the use of such calculation panels as NAFLD Fibrosis Score and Enhanced Liver Fibrosis (ELF). NFS (NAFLD Fibrosis Score) includes the analysis of six parameters (age, BMI, triglyceride levels, platelet count, albumin, AST to ALT). This scale has a $90 \%$ sensitivity and $60 \%$ specificity to exclude the diagnosis of liver fibrosis and $67 \%$ sensitivity and $97 \%$ specificity for its detection. ELF (Enhanced Liver Fibrosis) includes indicators such as GC, P3NP, TIMP-1, age. The developed algorithm allows to diagnose fibrosis with a sensitivity of $90 \%$ and exclude fibrosis with a negative predictive value of $92 \%$.

Non-invasive tests for fibrosis (FibroTest, ELF, and FibroMeter) can be used to differentiate between progressive and minimal/absent fibrosis, but some suggest the appropriate definition of the stage of fibrosis. The presence of these risk factors significantly limits the need for a biopsy. Some risk factors (age, BMI, type 2 diabetes, AST / ALT ratio) in combination with platelet count and serum albumin concentration are identified in the "fibrosis scale" with NAFLD, which allows accurately predict the pres- 
ence or absence of fibrosis in most patients. This scale has recently been combined with the European panel of serum markers of fibrosis, which has allowed differentiating various stages of fibrosis with an accuracy of over $90 \%[1 ; 12 ; 17]$.

Unfortunately, none of the available non-invasive methods are able to distinguish steatosis from NASH. This is very important, since the diagnosis of steatohepatitis determines patients at risk for developing fibrosis and justifies more intensive recommendations for lifestyle changes and the use of pharmacological treatments. Therefore, the "gold standard" for the diagnosis of NASH is still biopsy of the liver, as it is the only reliable method that allows to diagnose the inflammation and fibrosis. However, the result depends on the location of the biopsy. Two aspects are taken into account, when diagnosing the NAFLD: the level of fibrosis and the level of inflammation [3].

Unfortunately, none of the available non-invasive laboratory or visualization methods are able to distinguish steatosis from NASH. From a clinical point of view, this is very important, since the diagnosis of steatohepatitis determines patients at risk for developing fibrosis and justifies more intensive recommendations for lifestyle changes and the use of pharmacological treatments. Therefore, the "gold standard" for the diagnosis of NASH is still biopsy of the liver, as it is the only reliable method that allows you to evaluate inflammation and fibrosis. However, the result largely depends on the location of the biopsy in connection with the uneven spread of fibrosis. When establishing a diagnosis for the diagnosis of NAFLD, two aspects are taken into account: the level of fibrosis and the level of inflammation [3].

The waist circumference, the liver obesity index (IOP), and lipid accumulation products is closely related to the IR, indicate liver steatosis and may be useful for large screening among the general population instead of ultrasound. The HOMA and QUICKI indices are most commonly used by surrogate IR or insulin sensitivity indexes. Other, more sophisticated methods are based on measuring glucose and insulin levels in the test for glucose tolerance. Insulin sensitivity can also be quickly evaluated in the study of the lipid spectrum by calculating the ratio between triglycerides and high-density lipoprotein cholesterol (HDL). SteatoTest and the NSCLC scale have a higher sensitivity than ultrasound and can be used to quantify steatosis. Only SteatoTest and IOP are independently validated [11]. 
Treatment. At present, there is no specific therapy for NAFLD in combination with obesity. Meanwhile, steps were taken to form recommendations for the correction of fat and carbohydrate metabolism in general.

Directions of treatment that have a positive effect on patients with NAFLD and obesity:

- nutrition correction, carried out under the supervision of a nutritionist and a psychologist, taking into account the physical activity of the patient;

- Aerobic exercise (walking for 30 minutes daily, or $5 \mathrm{~km}$ a day three times a week);

- Reducing the number of calories consumed to $<30 \mathrm{kcal} / \mathrm{kg}$ per day and keeping the diet low in saturated and transgenic and simple carbohydrates;

- gradual loss of body weight (10\% body weight over six months), since rapid weight loss ( $>1.6 \mathrm{~kg}$ per week) can contribute to the progression of NAFLD;

- Correction of concomitant diseases such as diabetes mellitus, obesity, and metabolic syndrome;

- Patients with abnormal obesity (body mass index $>40$ ) may be recommended for bariatric surgery;

- the appointment of vitamin E (400-800 mg per day), vitamin D (1000 mg per day), the use of omega-3 fatty acids (fish oil) and omega-9 (olive oil);

- use of metformin / pioglitazone with ursodeoxycholic acid (15 mg / $\mathrm{kg}$ / day), if the index of insulin resistance (NOMA) $>2$.

Taking into account that oxidative stress is one of the components of the pathogenesis of NAFLD, the effect of vitamin E (tocopherol) is analyzed in the treatment. According to American recommendations for NAFLD, vitamin E, administered at a daily dose of 800, improves liver histology in patients without diabetes with histologically verified non-alcoholic steatohepatitis. It is emphasized that high doses of vitamin E can increase mortality from other causes $[3 ; 5 ; 7]$.

Among the drugs of choice is $\alpha$-lipoic acid, a coenzyme in the enzyme complex of pyruvate dehydrogenase, which is involved in the oxidative decarboxylation of pyruvic acid and $\alpha$-ketoacids. The drug inactivates free radicals and has a protective effect on the peroxide lipid oxidation in mitochondria and microsomes. According to the research, the use of $\alpha$-lipoic acid in the complex treatment of patients with NAFLD contributed to the improvement of lipid and carbohydrate metabolism, lowering the level of ALT $[5 ; 9]$. 
Most of the patients indicated therapy, which combines stabilize the membranes of hepatocytes, antioxidant protection, immune modulation, which provides anti-inflammatory and antifibrotychnu activity. To this end, pathogenetically justified use in the treatment of essential phospholipids NAFLD, ursodeoxycholic acid, and ademetionine, silymarin. Unfortunately, a large randomized controlled trial showed no benefits two years of use of UDCA (13-15 mg/ $/ \mathrm{kg}$ / day) in NASH, including its effect on liver histology. At the same time the more pronounced hepatoprotective effect of high-dose UDCA $(25-30 \mathrm{mg} / \mathrm{kg})$ and a new therapeutic agent - obetyholevoyi acid [6].

Given the fact that the key mechanism in the development of NAFLD and its complications in visceral obesity is the IR, the use of pharmacological agents that have a positive effect on the sensitivity of tissues to insulin, - insulin sensitizers, is confirmed. The most studied of these drugs is metformin, which not only directly affects the immune system but also has some beneficial metabolic effects. In the liver, metformin inhibits gluconeogenesis and glycogenolysis, as well as lipolysis and oxidation of free fatty acids, in skeletal muscle and adipose tissue, the drug stimulates tyrosine kinase activity of the insulin receptor, activates the glucose transporter GLUT-4, promotes increased absorption, utilization and oxidation of glucose, increases sensitivity to insulin In the adipose tissue, the drug suppresses lipolysis and oxidation of FFA. The most effective dose of metformin for the treatment of NAFLD, according to virtually all studies, is 1500-2000 mg per day, and the duration of treatment is at least one year. The duration of treatment is practically unlimited. To avoid the development of side effects, it is recommended to start therapy at a dose of $500 \mathrm{mg}$, increasing it gradually to a therapeutic dose of $500 \mathrm{mg}$ per week [9].

The influence of metformin on the histological picture of the liver according to literature is ambiguous. Some studies have shown that steatosis decreases on the background of treatment with metformin in patients with NAFLD, and, according to some reports, fibrosis of the liver. Instead, according to the recommendations of the American Association for the Study of Liver Diseases (AASLD, 2012), metformin therapy reduces the IR and the level of aminotransferases, but does not significantly affect the improvement of the histological picture in the liver, and is therefore not recommended for the treatment of NAFLD. Thus, the use of metformin for the treatment of NASH is currently considered as experimental [13]. 
Recently, data on the positive effect of thiazolidinediones (rosiglitazone and pioglitazone) that reduce IR over the course of NAFLD are given. Thus, the appointment of thiazolidinediones to patients with obesity and NAFLD improved the histological picture of the liver, reduced the risk of IR, reduced levels of transaminases in the blood. However, the use of thiazolidinediones may be accompanied by the development of undesirable effects, such as an increase in body weight due to fat accumulation in the subcutaneous fatty tissue, moderate anemia as a result of an increase in the volume of circulating blood, due to which the use of drugs in this group is limited. Pioglitazone should be administered with extreme caution to patients with heart disease (a characteristic concomitant pathology for NAFLD patients), and ECG should always be performed before a decision about the course of treatment $[3 ; 5 ; 7]$.

An important place in the treatment of NAFLD is to correct dyslipidemia to reduce cardiovascular risk, including using hypolipidemic drugs. In practice, there are often concerns associated with increased levels of transaminases against statin therapy, but results of studies indicate the efficacy and safety of the use of statins in NAFLD. According to researches, in patients, regardless of the presence of obesity and NAFLD, increased activity of transaminases, in the background of taking statins, is observed in $0.5-2 \%$ of cases, depends on the dose of drugs and is temporary. When combined with NAFLD and hypertriglyceridemia, the omega-3 polyunsaturated fatty acids prescription is possible [10].

Since, there are no specific drugs for the treatment of NAFLD, the study of the intestinal microbiota influence on the pathogenesis and progression of this disease may have new therapeutic perspectives. In particular, the modulation of the intestinal microbiota can be achieved by the use of prebiotics, probiotics, selective intestinal antibiotics (rifaximin), and fecal microbiota transplantation. The use of prebiotics increases the production of propionate, which suppresses lipogenesis in the liver. The addition of prebiotic fibers provided a lipid-lowering effect in animal studies, presumably by reducing lipogenesis due to a decrease in the activity of enzymes responsible for the esterification of free fatty acids with the formation of new triglycerides. Other studies have shown that Bifidobacterium longum, along with lifestyle modification, reduces cytotoxic activity, plasma lipid levels, insulin resistance index (NOMA-index) and NASH activity index in a small sample of patients in whom the disease has been confirmed by biopsy [16]. 
The overall annual incidence of hepatocellular carcinoma (HCC) in patients with cirrhosis on the background of NASH is $2.6 \%$ per year. Consequently, control of abdominal ultrasound in such patients should be performed every six months. Although ultrasound can be technically difficult to implement in obese patients, there is currently no evidence to support routine use to monitor computer or magnetic resonance imaging. Although a tumor biomarker such as $\alpha$-fetoprotein has recently been excluded from European Clinical Recommendations, it is still widely used in clinical practice along with ultrasound scans every six months. It is known that there is a clear link between obesity and malignancy, and hyperinsulinemia is well known as an independent risk factor for many tumors $[7 ; 13 ; 15]$.

Aberrant genes involved in metabolic pathways, such as the pathway of AMP-activated protein kinase (AMPK) -LKB1, is now considered as therapeutic targets in the treatment of cancer. Metformin acts by suppressing the production of liver glucose through the LKB1-AMPK-mediator mechanism. There are also data that reduce the risk of HCC and statins. It was found that those who received statins were less likely to develop fecal colitis (corrected HS 0.63, 95\% CI 0.52: 0.76). The most significant reduction in risk was noted in the Asian population, although it was also observed in western populations $[1 ; 6 ; 12 ; 14]$.

Despite numerous clinical trials, there is currently no specific NSAID therapy, but many studies are underway, and new drugs are being developed that increase the life span of hepatocytes: oral peroxidase inhibitors, phosphodiesterase inhibitors, recombinant leptin, and adiponectin.

\section{Conclusions}

The notion of NAFLD in recent years has been enriched with scientific evidence that emphasizes its important role in the spectrum of diseases. In particular, it was found that NAFLD is the most common variant of liver damage and is responsible for the development of a significant proportion of fatal complications. No less important is the association of NAFLD with some metabolic disorders that are characteristic of obesity, diabetes mellitus and cardiovascular disease. Similar etiologies and pathogenetic mechanisms of this group of diseases determine the approaches to treatment. Lifestyle modification recommendations and some medicines used to treat diabetes, obesity, and dyslipidemia are useful for normalizing the morphological and functional features of the liver. 


\section{References:}

1. Angulo P, Keach JC, Batts KP et al. Independed predictors of liver fibrosis in patients whis nonalcoholic steatohepatitis. Hepatology. 1999;30:1356-1362.

2. Bugianesi E, Gastaldelli A, Vanni E et al. Insulin resistance in non-diabetic patients with non-alcoholic fatty liver disease: sites and mechanisms. Diabetogia. 2005;48:634-642.

3. Chalasani N, Younossi Z, Lavine J et al The Diagnosis and Management of Non-Alcoholic Fatty Liver Disease: Practice Guideline by the American Association for the Study of Liver Diseases, American College of Gastroenterology, and the American Gastroenterological Association. Hepatology. 2012;55(6):2005-2023.

4. Clark JM. The prevalence and etiology of elevated aminotransferase levels in the United States. Am J Gastroenterol. 2003;98:955-956.

5. Diehl AM, Day C. Cause, Pathogenesis, and Treatment of Nonalcoholic Steatohepatitis. N Engl J Med. 2017. -Vol. 377(21). - P.2063-2072

6. Engin A. Non-Alcoholic Fatty Liver Disease. Adv Exp Med Biol. 2017;960:443-467

7. Huang MA, Greenson JK, Chao CC. One-year intense nutritional counseling results in histological improvement in patients with non-alcoholic steatohepatitis: a pilot study. Am J Gastroenterol. 2005;100:1072-1081.

8. Kitade H, Chen G, Ni Y. Nonalcoholic Fatty Liver Disease and Insulin Resistance: New Insights and Potential New Treatments. Nutrients. 2017;9(4)

9. Lazaridis $\mathrm{N}$, Tsochatzis E. Current and future treatment options in non-alcoholic steatohepatitis (NASH). Expert Rev Gastroenterol Hepatol. 2017 Apr;11(4):357-369

10. Liyanagedera S, Williams RP, Veraldi S. The pharmacological management of NAFLD in children and adolescents. Expert Rev Clin Pharmacol. 2017;10(11):1225-1237.

11. Neuschwander-Tetri BA. Non-alcoholic fatty liver disease. BMC Med. 2017;15(1):45

12. Nonalcoholic Fatty Liver Disease and Nonalcoholic Steatohepatitis. World Gastroenterology Organisation Global Guidelines. June 2012. http://www.worldgastroenterology.org/NAFLD-NASH.

13. Obesity. World Gastroenterology Organization Global Guideline. 2009. http://www.worldgastroenterology.org/obesity.

14. Pár A, Pár G. Advances in the pathogenesis of non alcoholic fatty liver disease. Orv Hetil. 2017;158(23):882-894

15. Reccia I, Kumar J, Akladios C. Non-alcoholic fatty liver disease: A sign of systemic disease. Metabolism. 2017;72:94-108

16. Sidhu M, Poorten D. The gut microbiome. Aust Fam Physician. 2017;46(4):206-211.

17. Sookoian S, Pirola CJ. Systematic review with meta-analysis: risk factors for non-alcoholic fatty liver disease suggest a shared altered metabolic and cardiovascular profile between lean and obese patients. Aliment Pharmacol Ther. 2017;46(2):85-95

18. Stefan N, Kantartzis K, Häring H. Causes and Metabolic Consequences of Fatty Liver. Endocrine Reviews. 2008;29(7):939-960. 
19. Williams CD, Stengel J, Asike MI, et al. Prevalence of nonalcoholic fatty liver disease and nonalcoholic steatohepatitis among a largely middle-aged population utilizing ultrasound and liver biopsy: a prospective study. Gastroenterology 2011; 140(1): 124-31.

20. Younossi M Z. Changes in the Prevalence of the Most Common Causes of Chronic Liver Diseases in the United States From 1988 to 2008. Clinical Gastroenterology and Hepatology 2011;6:524-530.

21. Yoneda M., Naka S., Nakano K. Involvement of a periodontal pathogen, Porphyromonas gingivalis on the pathogenesis of non-alcoholic fatty liver disease. BMC Gastroenterol. 2012; 12: 16. 